\title{
Voice Over Internet Protocol (VoIP) And One University Application
}

Victoria L. Figiel

Mike Whitlock, (E-mail: whitlock@ troyst.edu), Nova Southern University

\begin{abstract}
Internet telephony is emerging as an inexpensive alternative to the high costs of long distance and transcontinental calls. Colleges and universities may also benefit from this technology shift. This paper addresses the impact of new Voice over Internet Protocol (VoIP) technologies and the business conditions moving consumers to consider the new technologies. Two models: technology push and market pull, identify the stakeholders in the move to VoIP technologies. The models are discussed and applied to the needs of a college campus providing traditional and nontraditional education delivery methods.
\end{abstract}

\section{INTRODUCTION}

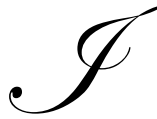

nternet telephony is emerging as an inexpensive alternative to the high costs of long distance and transcontinental telephone calls (Yeo, Hui, Soon, \& Ang, 2003). For companies currently spending approximately three percent of their gross sales on local and long distance telecommunications services (Harte, 2003), cheaper alternatives can have a significant impact to the bottom line. Internet telephony also promises costs savings and new revenue sources for some service providers (Sharafeddine, Riedl, Glasmann \& Totzke, 2003). According to Isenberg (p. 38, 2003), phone companies still make more money from voice than from other network traffic, even though the volume of data traffic now exceeds voice. Furthermore, Internet voice is getting better and better - its quality can, in fact, far exceed the "toll quality" voice of plain old telephone service (POTS).

This paper addresses the impact of new Voice over Internet Protocol (VoIP) technologies. Specifically under review are the business conditions moving consumers to consider telephony and data options, the current availability of VoIP options, and the foreseeable future of the VoIP technologies from the perspective of business stakeholders. In order to review the impact in an actual business application, the cases of VoIP alternatives are applied to higher education satellite campus telephony and data needs.

The satellite campus studied offers conditions of various types that can be generalized to many other university, college and business applications. For example, they have several campus settings, and a large contingent of non-traditional on-line students from areas of the world including students in North America, Africa, Europe and Asia. This institution's professors must be available through various electronic means at different times to meet the students' needs. Remote working applications are also an issue. Finally, a VoIP solution for this campus is offered from which other businesses and universities may find similarities and applications for their specific case.

\section{CURRENT BUSINESS CONDITIONS REGARDING TELEPHONY AND DATA}

The price of long distance telecommunications is driving the move to cheaper alternatives (Zubey, Wagner, \& Otto, 2002). VoIP is one of those options. Using Net2Phone VoIP service, a call from the U.S. to Mexico costing $\$ 2.00$ per month and $\$ 0.59$ per minute with AT\&T (AT\&T Consumer, 2004), costs $\$ 0.149$ per minute on their network (Net2Phone Corporation, 2004).

Still, there are several disadvantages to using VoIP today, and some that users may find unacceptable. Quality of service (QoS), in some cases, does not meet voice grade quality transmission (Zubey, Wagner, \& Otto, 2002). 
Transmission lines must meet higher levels of quality for Internet calls. For these reasons, Gaynor (2003) suggests that end users, and network owners alike, would benefit from applying Options Theory to the case of VoIP and purchase decisions. He says that like the uncertain environment of options trading, new communications alternatives should follow a model of valuing choices from many solutions. Try it before buying it. Experimentation with various services and features lead to a better understanding of needs and capabilities before purchase decisions are made.

\section{STAKEHOLDERS DRIVING THE NEW TECHNOLOGY}

Heron and Warren (2001) define four stakeholders in the move to new telecommunications technologies, especially VoIP: service providers, international standards organizations, manufacturers, and end users. The service providers' main mission is to build economic networks for the end users. In particular, service providers must leverage their capital investments with minimum recurring costs. The international standards organizations are pictured by Heron and Warren as the independent evaluator of components. Objective evaluation is this group's primary purpose. Manufacturers and end users are the other stakeholders. While manufacturers in this scenario are not product drivers per se, they are defined as reactive to the service provider and the end user's need for new technologies that work with other manufacturers products. For this reason, Heron and Warren call this the technology push scenario.

Heron and Warren (2001) also define an end user initiated model for new telecommunications development: the market pull model. The market pull model is described as a commercial model. This model shows how the market requirements are taken through a process of recognizing a market need, developing technical requirements, issuing a request for information, a responding to the invitation to tender, contracting with a manufacturer, and finally delivering the product. The authors stress the need to balance technology assessments with both the technology push and market pull models.

Since VoIP uses the Internet to replace traditional phone service, several companies want to cash in on the money now going to the traditional telephone companies (Lashinsky, 2004). This includes cable companies, traditional telephone companies hoping to hold on to the revenue, and companies purely designed to sell VoIP services; nicknamed "pure plays". Pure plays include the likes of Net2Phone, whose focus is in selling wholesale VoIP service; Vonage, with their national VoIP service with over 97,000 subscribers; and 8X8 out of Santa Clara, California, offering local and long distance calling in the U.S. and Canada for less than twenty dollars per month. Other pure plays include Dialpad.com and PhoneFree.com (Zubey, Wagner, \& Otto (2002).

Net2Phone advertises long distance service with VoIP as low as four cents per minute to free if the user will accept banner advertisements. Cable companies like Time Warner Cable, Cox Communications, and Cablevision Systems have only recently become sellers of VoIP and are still in relatively few markets. The traditional telephone companies like AT\&T, BellSouth and Verizon are also rolling out VoIP. Verizon announced plans to build the nation's largest network for VoIP and other Internet services.

\section{CURRENT CONDITIONS AT THE STUDIED CAMPUS}

The satellite campus is a self-contained campus providing student services, academic instruction, and technology support. Student records, library services, and financial support systems such as payroll, accounts receivable, and accounts payable connect to the host university's network. The satellite campus provides academic support to ten other smaller sites.

The satellite campus primarily serves non-traditional students. Many are adults who require night, weekend, or distance programs because of jobs or family commitments. Some students are military residing in almost every time zone around the world. Distance programs are a popular option along with weeknight and weekend formats. The learning platform used for distance programs is Blackboard. Many of the instructors teaching in the classroom enhance their instruction with Blackboard. Due to the academic oversight provided by the host institution and accreditation pressures, the satellite campus is constantly looking for ways to enhance the quality of distance courses with particular emphasis on student-instructor interaction. 
Instructors using Blackboard are required to be available to students on a daily basis. The goal is to respond to all student questions within 24 hours. Student evaluations indicate that most instructors respond at least that fast, but there are exceptions. Several software applications are being considered to enhance the quality of distance education. These include adding voice and video to course sites. For classroom instruction, technology linking classrooms at different locations, allowing a single instructor to teach multiple locations simultaneously, is also being explored.

Coordination of staff and faculty activities between supported sites is handled by traditional voice communications and e-mail. Training usually requires those being trained or the trainer to travel. This is further complicated when multiple sites are involved. Training resources are inadequate to cover all remote locations effectively.

The satellite campus uses a Cisco PBX. This PBX ties the other locations to the satellite campus through trunks. In total, there are over 125 separate phone extensions on the system including all sites. The main PBX is connected to another PBX at the main host institution's campus through additional trunks.

One additional note of importance on the current condition is that the leadership and administration at both the satellite campus and the host institution are supportive of any new technology that enhances distance education and reduces expenses.

\section{VoIP TECHNOLOGY TODAY}

\section{Current Business Telephony}

Many businesses today have private telephone systems that may be owned, leased or managed internally or externally. The private telephone systems can consist of inexpensive options to sophisticated integrated voice and data networks using local area networks (LAN), or private branch exchange (PBX) systems (Harte, 2003). The LAN and PBX systems provide for switching between incoming multiple channel lines, called trunks, yet also provide for advanced calling features shared by the users of the LAN or PBX. These communications alternatives are provided by many companies at various different price points, and have been in place for several decades. But first, before examining the use of new technology, it is advisable to understand current network access methodology.

Whether considering traditional telephony, or the newest converged telephone and data networks, and regardless of whether privately owned or publicly leased, the architecture can be thought of as three main elements (Ohrtman, 2003) each with a price tag for the business using the service, or by default passed to them by the service provider. The three elements are the following: access, or the means of gaining access to the network, like a phone or personal computer; switching, or the way a call is controlled through the network to the other end; and transport, or the physical means of moving a call from one point to another.

\section{Access}

The telephone handset is the most common form of access technology today. This is true for a land-based system or cellular system (Ohrtman, 2003). From the perspective of the telephone companies, the access mediums are most often owned by the business and are generally known as customer premise equipment (CPE). Businesses may also use computers for access methodology today. In historical telephone systems, lines were purchased for each access methodology to be used simultaneously. That is not the case today, and can result in savings to the business. Voice and data may simultaneously share a communications link.

\section{Switching}

Switching is the means of controlling a call through the network (Ohrtman, 2003). In any network with more than just two points, and whether private or public, the switching elements contain the intelligence to send a call, or data transmission, in a selected path to reach the destination. Switching technology has evolved from a manual system 
to analog to digital systems with efficiencies gained in reliability, scalability, signaling, quality of service (QoS) and features. Many features that businesses employ are provided to them through the software in the switching computers.

\section{Transport}

The transport medium is the third element in the system (Ohrtman, 2003). It involves the means of moving the call, or data, from one point to another. Transport can be over copper wires, or fiber optics, or microwave. Each method offers various speeds for getting from one place to another at various reliability rates and therefore, various price points. From the telephone company's perspective, transporting the maximum number of conversations, or channels, over one pipe represented a significant means of cutting cost. Hardware that could multiplex more and more channels onto one transport medium has grown significantly over the past thirty years. From the one wire, one conversation of yesterday, to common optical carrier (OC) levels like OC-12 and OC-48 carrying over 8,000 and 32,000 channels, respectively, transport has undergone a major technology shift.

Access, switching and transport remain important elements even in the newest technologies. The elements are discussed from the perspective of VoIP applications next.

\section{VoIP and Its Elements}

Access to the VoIP network can be accomplished as phone-to-phone, phone-to-personal computer, or personal computer-to-personal computer (Ohrtman, 2003). PingTel and e-tel were early adopters of the new VoIP technology and produced a personal computer in a handset for use with VoIP. Other access connections can be made through specialized personal computers that support connections of IP enabled phones via an ordinary telephone jack type outlet. The business user should be aware that this technology is new and IP phones may cost $\$ 500$ or more as compared to the traditional PBX phone at about $\$ 150$ each. These costs can be offset by the business user in features and long distance charges.

The switching elements in VoIP are servers and gateways. Computer servers and gateways are the primary components in a VoIP network (Harte, 2003). The purpose of the server is to control the system access and call processing. The gateways are the hardware components that convert the packet information to signals usable by the access devices.

Pricing considerations are also important for the switching elements: servers and gateways. This is not generally an issue for the business executive using VoIP, but more for the telecommunications provider. These elements are scalable from small residential applications of two to eight ports to carrier-grade gateways that can switch high density trunks (Ohrtman, 2003).

Transport is the reason VoIP exists (Ohrtman, 2003). Long distance service providers wanted a more economical means of passing information, and that is by using the IP network, which is available ubiquitously. As the long distance carriers found economies, they passed those on to their customers. The savings passed on from long distance service providers is what makes VoIP attractive to businesses.

\section{VoIP and Convergence}

"The dominant trends in the telecommunications industry today are convergence in the areas of technologies, transport and access networks, and services" (Dianda, Ho, \& Kocan, 2000, p. 56). Lago (2002, p. 2) defines convergence as "the possibility of transparently supporting any communication type, over any type of network and terminal."

The benefits of convergence and an Internet Protocol network solution can be summarized as follows (Das, Lee, Basu, \& Sen, 2003): 
- $\quad$ cost reduction, converging voice and data traffic improves network efficiency and lowers operations costs;

- $\quad$ simplification, which leads to standardization, and therefore, convergence of different functions on the same technology;

- $\quad$ consolidation, allowing the service provider to eliminate points of failure by combining operational units, and even the ability to combine accounting systems; and

- $\quad$ advanced applications, such as multimedia and multiservice applications.

\section{VoIP and Reliability}

VoIP relies on a packet-based network, meaning that information; one voice call, or data transmission, is sent in discrete packages of information. The packets may each traverse the network differently and then be reassembled in correct order for the receiver to hear the correct voice, or receive the correct data transmission. Since the packets can take different paths to the called party's location, it is often said that if a segment of the network experiences an outage, then that packet will take another path to the destination (Levendel, 2002). This is an advantage of VoIP over the historical means of transporting voice and data. Previously, if a circuit went out of service, then communications to that called location could be delayed until the connection was repaired or new routing of calls was executed. It is true that improvements have been made in traditional networks with self-healing fiber rings. Still, VoIP does offer some additional measure of reliability given the parameters discussed previously, but that does not take into account other points of failure.

Reliability of the VoIP networks is affected by various network designs related to the three network elements previously mentioned. For example, the switching element is a critical component to that end. Service providers must be able to not only observe the switch elements but must be able to control them through effective operational management (Levendel, 2002). There is yet to be a standardized failure management strategy. Other points include issues with software and access technologies and transmission.

\section{POTENTIAL APPLICATIONS AT THE SATELLITE CAMPUS}

Cost savings, portability, integration of audio and video in delivery of distance instruction, and transparency to students regarding instructor locations are all benefits that can flow from moving to VoIP at the satellite campus. There would be start-up costs involved in acquiring a VoIP LAN and over 125 VoIP compatible phones or compatible personal computers. Still, the VoIP application could enhance many of the ways instructors and students communicate. Interaction between students and instructors is a major accreditation concern.

One example where VoIP can improve the status quo is a case in which a professor is delivering his course to more than one location. The professor is in one location for part of the term and in the other location for part of the term. With VoIP and a VoIP compatible phone, the instructor can move between the various locations and the LAN will register him as a user of the system such that any one dialing his number will reach him regardless of location. Voice mail and other features are transparent to the move and follow him as well. If the professor should choose to move to another site within the VoIP cluster, the calls will follow him there as well. Even if he should decide to leave the cluster locations, he can dial-in to the LAN and receive calls at his number as if at any of the work locations.

\section{CONCLUSIONS}

This paper addressed the impact of new Voice over Internet Protocol (VoIP) technologies. Specifically under review were the business conditions moving consumers to consider telephony and data options, the current availability of VoIP options, and the foreseeable future of the VoIP technologies from the perspective of business stakeholders. In order to review the impact in an actual business application, the cases of VoIP alternatives were applied to the telephony and data needs on higher education satellite campus arrangement. 


\section{SUGGESTIONS FOR FUTURE RESEARCH AND GENERALIZATION TO OTHER BUSINESSES}

This paper addressed the impact of new VoIP technologies specifically to the case of a university setting. While this particular satellite campus offered various scenarios for VoIP application, these can also be generalized to many other business applications. For example, businesses with remote workers, or multiple locations will find efficiencies and effectiveness with VoIP solutions. Finally, a VoIP solution for this satellite campus was offered.

Future research should review the cost implications and technology needs for non-traditional business arrangements. Those that may find benefit in this type of VoIP environment include businesses with remotely located workers or traveling workers. New architectures to meet the needs of these business groups may benefit from building on the VoIP backbone described in this article.

\section{REFERENCES}

1. AT\&T Consumer. (n.d.). AT\&T Global. Retrieved February 5, 2004, from http://www.consumer.att.com/ global/english

2. Das, S. K., Lee, E., Basu, K., \& Sen, S. K. (2003). Performance optimization of VoIP calls over wireless links using H.323 protocol. IEEE Transactions on Computers, 52(6), 742-752.

3. Dianda, J. R., Ho, B. W., \& Kocan, K. F. (2000). Reducing complexity for converged voice/data networks and services architecture. Bell Labs Technical Journal, April/June, 55-71.

4. Gaynor, M. (2003). Linking market uncertainty to VoIP service architectures. IEEE Internet Computing, July/August, 16-22.

5. Harte, L. (2003). Voice over data networks for managers. Fuquay-Varina, NC: Althos Publishing.

6. Heron, A. J., \& Warren, A. H. (2001). Evaluating VoIP technology: Technology push/market pull. BT Technology Journal, 19(2), 158-167.

7. $\quad$ Isenberg, D. S. (2003). The end of the middle. IEEE Spectrum, (January), 37-38.

8. Lago, P. (2002). A policy-based approach to personalization of communication over converged networks. Proceedings of the Third International Workshop on Policies for Distributed Systems and Networks (POLICY'02), 0-7695-1611-4/02.

9. Lashinsky, A. (2004). Leaping into the VoIP. Fortune, February 9, 68.

10. Levendel, I. (2002). There is more to service carrier-grade quality than Quality of Service (QoS), or has George Washington been telling the truth? Proceedings of the IEEE International Symposium on Network Computing and Applications (NCA'01), 0-7695-1432-4/02.

11. Net2Phone Corporation. (2004). Net2Phone - the home of Internet telephony at Retreived February 5, 2004 from http://www.net2phone.com/consumer/commcenter/rates.asp\#

12. Ohrtman, Jr., F. D. (2003). Softswitch: Architecture for VoIP. New York: McGraw-Hill.

13. Sharafeddine, S., Riedl, A., Glasmann, J., \& Totzke, J. (2003). On traffic characteristics and bandwidth requirements of voice over IP applications. Proceedings of the 8th IEEE International Symposium on Computers and Communication (ISCC'03), 1530-1346/03.

14. Yeo, C. K., Hui, S. C., Soon, I. Y., \& Ang, L. M. (2003). H.323 compliant voice over IP system. International Journal of Computer Applications in Technology, 16(4), 143-153.

15. Zubey, M. L., Wagner, W., \& Otto, J. R. (2002). A conjoint analysis of voice over IP attributes. Internet Research: Electronic Network Applications and Policy, 12(1), 7-15. 